\title{
Factores psicosociales, estrés psicológico y burnout en enfermería: un modelo de trayectorias
}

\section{Psychosocial factors, psychological stress, and burnout in nursing: a model of trajectories}

\section{Fatores psicossociais, estresse psicológico e burnout em enfermagem: um modelo de trajetórias.}

\author{
J.F. Brito-Ortíz ${ }^{\mathrm{a} 1}$, A. Juárez-García ${ }^{\mathrm{b} 2}$, M.E. Nava-Gómez ${ }^{\text {3** }}$, J.J. Castillo-Pérez ${ }^{\mathrm{d} 4}$, E. Brito-Nava ${ }^{\mathrm{e}}$ \\ ORCID: \\ a $0000-0002-9435-1711$ \\ b $0000-0003-3264-679 X$ \\ c $0000-0003-2000-8520$ \\ d $0000-0002-3725-3541$ \\ e 0000-0001-7298-3438 \\ ${ }^{1}$ Escuela de Ciencias de la Salud, Universidad Autónoma de Baja California, Tijuana, Baja California, México. \\ ${ }^{2}$ Centro de Investigación Transdisciplinar en Psicología, Universidad Autónoma del Estado de Morelos, Cuerna- \\ vaca, Morelos, México \\ ${ }^{3}$ Licenciatura en Enfermería, Universidad Autónoma de Baja California, Tijuana, Baja. California, México \\ ${ }^{4}$ Coordinación de Investigación en Salud, División de Evaluación de la Investigación, Instituto Mexicano del \\ Seguro Social, Ciudad de México, México \\ ${ }^{5}$ Hospital Universitario, Benemérita Universidad Autónoma de Puebla, Puebla., México
}

Recibido: 11 mayo 2018

Aceptado: 13 enero 2019

\section{Resumen}

Introducción: El personal de enfermería se enfrenta cotidianamente a diferentes eventos asociados al estrés, factores psicosociales y otros mecanismos que afectan su salud mental.

Objetivo: Evaluar la correlación entre la demanda psicológica, el control laboral, el apoyo social, el estrés psicológico, y las dimensiones del Síndrome de Burnout (ilusión por el trabajo, desgaste psíquico, indolencia y culpa), en un modelo de trayectorias en enfermeras mexicanas.

Metodología: Estudio transversal, observacional, analítico, conducido en 357 profesionales de enfermería de Morelos, México, seleccionados por conveniencia. Los datos fueron colectados empleando tres

* Autor para correspondencia. Correo electrónico: martha.nava40@uabc.edu.mx 
cuestionarios, la Escala de Estrés Percibido, el Cuestionario del Contenido del Trabajo y el Cuestionario para la Evaluación del Síndrome de Quemarse por el Trabajo. Los programas SPSS 19 y LISREL 8.3 fueron empleados para el procesamiento y modelaje de los datos.

Resultados: En el modelo de trayectorias, se especificó como variable mediadora de los factores psicosociales y de las dimensiones del Síndrome de Quemarse por el Trabajo (SQT), al estrés psicológico. Los índices de ajuste fueron aceptables. Se encontraron altos niveles de apoyo social, control laboral e ilusión por el trabajo; grados moderados de demanda psicológica y estrés psicológico; y bajos niveles de desgaste psíquico, indolencia y culpa.

Conclusión: Implicaciones para la disciplina, se propone implementar estrategias de intervención efectivas para mantener en el personal de enfermería bajos niveles de demandas psicológicas, altos niveles de control laboral, altos niveles de apoyo social, y niveles moderados de estrés, para prevenir, el SQT.

Palabras clave: Agotamiento profesional/enfermería; estrés psicológico; estrés laboral; México.

\section{Abstract}

Introduction: Nurses frequently face different events associated with stress, psychosocial factors, and other issues which have impacts on their mental health.

Objective: To assess the correlation between psychological demand, work control, social support, psychological stress, and burnout dimensions (illusion for the activity, psychic wear, indolence, guilt), within a model of trajectories among Mexican nurses.

Methodology: This is a transversal, observational, and analytical study conducted on a convenience sample of 357 nursing professionals from the state of Morelos, Mexico. Data were gathered using three questionnaires, the Perceived Stress Scale, the Job Content Questionnaire, y el Maslach Burnout Inventory. SPSS 19 and LISREL 8.3 were used to process and model the data.

Results: Psychological Stress was specified as the mediating variable of the psychosocial factors and dimensions of burnout within the model of trajectories. Adjustment indexes were acceptable. High levels of social support, work control, and illusion for the activity were found. Moderate degrees of psychological demand and psychological stress were found. Low levels of psychic wear, indolence, and guilt were found.

Conclusion: It is recommended to implement effective intervention strategies to maintain nursing personnel within low levels of psychological demand, high levels of work control, high levels of social support, and moderate levels of stress, all these in order to prevent the Syndrome of Burnout.

Keywords: Burnout, professional/ nursing; stress, psychological; occupational stress; Mexico.

\section{Resumo}

Introdução: O pessoal de enfermagem se enfrenta cotidianamente a diferentes eventos associados ao estresse, fatores psicossociais e outros mecanismos que afetam sua saúde mental.

Objetivo: Avaliar a correlação entre a demanda psicológica, o controle laboral, o apoio social, o estresse psicológico, e as dimensões e da Síndrome de Burnout (ilusão pelo trabalho, desgaste psíquico, indolência e culpa), em um modelo de trajetórias em enfermeiras mexicanas.

Metodologia: Estudo transversal, observacional, analítico, conduzido em 357 profissionais de enfermagem de Morelos, México, selecionados por conveniência. Os dados foram coletados empregando três questionários, a Escala de Estresse Percebido, o Questionário do Conteúdo do Trabalho e o Questionário para a Avaliação da Síndrome de Burnout no Trabalho. Os programas SPSS 19 e LISREL 8.3 foram empregados para o processamento e modelagem dos dados.

Resultados: No modelo de trajetórias, especificou-se como variável mediadora dos fatores psicossociais e das dimensões da Síndrome de Burnout no Trabalho (SQT), ao estresse psicológico. Os índices de ajuste foram aceitáveis. Encontraram-se altos níveis de apoio social, controle laboral e ilusão pelo trabalho; 
graus moderados de demanda psicológica e estresse psicológico; e baixos níveis de desgaste psíquico, indolência e culpa.

Conclusão: Implicações para a disciplina, propõe-se implementar estratégias de intervenção efetivas para manter no pessoal de enfermagem baixos níveis de demandas psicológicas, altos níveis de controle laboral, altos níveis de apoio social, e níveis moderados de estresse, para prevenir, o SQT.

Palavras chave: Esgotamento profissional/enfermagem; estresse psicológico; estresse ocupacional; México.

\section{Introducción}

El estudio del estrés psicológico, de los factores psicosociales y de las dimensiones del burnout en profesionales de enfermería, presenta un gran interés debido a que este personal es un grupo especialmente vulnerable a los efectos del estrés ${ }^{1,2}$ cabe mencionar, que el estrés crónico afecta en el personal de enfermería su salud mental y desempeño en el trabajo ${ }^{3,4}$.

El tema del estrés ha atraído la atención desde la publicación del libro The stress of life, en 1956, escrito por Selye, quien afirma que el estrés tiene conexión con aspectos biológicos, psicológicos y sociales. Posteriormente, diversas investigaciones han contribuido en forma paulatina a precisar la influencia de diferentes factores, Cooper ${ }^{5}$ analiza algunas de estas, donde se plantea que el estrés parece deberse al volumen del trabajo, a la responsabilidad respecto de otras personas, a la complejidad de las tareas y a una escasa delegación de funciones y toma de decisiones, esto sugiere que existe una asociación entre diversos factores psicosociales y el estrés; estos resultados han brindado los fundamentos para que diversos organismos, entre ellos la OIT, los incorporen a sus normativas.

Los factores psicosociales laborales son condiciones de trabajo que pueden ser positivas (factores protectores), es decir: facilitan el trabajo, el desarrollo de las competencias profesionales, además generan altos niveles de satisfacción laboral y motivación en los empleados ${ }^{6}$.

Los factores psicosociales pueden también ser negativos (factores de riesgo) y representan una fuente de niveles de estrés elevados ${ }^{7,8}$; el impacto de estos factores no se traduce de manera directa e inmediata en efectos adversos para la salud, sino que estos niveles altos de estrés juegan un rol esencial en los mecanismos de afectación. Aunque, se ha señalado que los factores de riesgo psicosociales poseen un mecanismo de afectación directa a la salud ${ }^{9}$, no existen estudios que expliquen mecanismos alternos al estrés, por lo que implícitamente se pueden considerar a los factores de riesgo psicosociales como estresores laborales ${ }^{10}$.

No obstante, la lista de factores de riesgo psicosociales a los que se expone el sector de enfermería puede ser amplia, destacan las variables que conforman el modelo de Karasek ${ }^{11}$, cuyas variables son las demandas del trabajo, el grado de autonomía o decisión (control) y el nivel de apoyo social (entre compañeros y con el jefe inmediato). Este modelo es el de mayor evidencia empírica en el estudio de los factores psicosociales correlacionados con el estrés laboral, y tiene amplias evidencias de su impacto en la salud del personal de enfermería ${ }^{12,13}$, inclusive al mexicano ${ }^{14,15}$.

La literatura asocia al Síndrome de Quemarse por el Trabajo (SQT) con una respuesta al estrés laboral crónico, que se presenta cuando las estrategias de afrontamiento fallan, por lo cual desencadenan y propician un efecto de factores psicosociales negativos ${ }^{16,17}$.

Gil-Monte ${ }^{18}$ desarrolló un cuestionario con cuatro dimensiones que integran este síndrome (1. baja ilusión por el trabajo: se refiere al deseo que tiene una persona por alcanzar las metas profesionales laborales; 2. alto desgaste psíquico: hace referencia al deterioro tanto emocional como físico del individuo; 3 . alta indolencia: especifica la presencia de actitudes negativas de indiferencia y cinismo; 4. culpa: se define como los sentimientos que surgen a raíz del comportamiento y las actitudes negativas desarrolladas por el profesional, que presta su trabajo hacia quienes lo reciben) y lo denominó CESQT, herramienta "adhoc" a la cultura hispanoamericana para la evaluación del fenómeno del burnout o Síndrome de Quemarse en el trabajo ${ }^{18}$. El SQT implica el desarrollo de actitudes negativas hacia el trabajo y pérdida de interés 
hacia los clientes o usuarios del servicio, este fenómeno altera el funcionamiento mental, origina una capacidad de toma de decisiones más lenta y menos segura, aumenta la ansiedad y reduce la autoestima, la confianza y el autocontrol. A largo plazo, conlleva insatisfacción, ejecución empobrecida y hasta el abandono de la actividad ${ }^{19,20}$.

Por lo tanto, tener en cuenta que el elevado nivel de apoyo social disminuye el estrés, que altos niveles de demandas psicológicas y el pobre control laboral lo aumentan, el personal de enfermería sufrirá tensión cuando estos dos últimos factores psicosociales sean crónicos $^{21,22}$; además si se considera que el SQT se define como una respuesta al estrés crónico, característico en profesionales de enfermería que trabajan en atención hacia las personas ${ }^{23}$, se propone un modelo de trayectorias que analiza la relación que guardan estos factores psicosociales, el estrés psicológico y el SQT, en donde el estrés es el mediador del impacto de los primeros en el SQT.

Las instituciones que ofertan servicios de salud se caracterizan por recibir y generar, entre sus trabajadores, altas demandas emocionales y psicosociales que son potenciales fuentes de estrés. De este modo, resulta relevante conocer la prevalencia de los factores psicosociales en el trabajo, la demanda psicológica, el control en el trabajo y el apoyo social; así como conocer la prevalencia del estrés percibido, e identificar si el personal de enfermería presenta el SQT.

Un estudio sobre estrés y Síndrome de Burnout en personal de enfermería de un hospital materno infantil de atención a la población abierta, mostró que ante niveles bajos de estrés en este personal, no hubo manifestación de síntomas de burnout ${ }^{24}$; el estrés presentó correlaciones estadísticamente significativas con las dimensiones del burnout, positiva con el cansancio emocional $(\mathrm{r}=0.231)$, positiva con la despersonalización $(\mathrm{r}=0.236)$ y negativa con la realización personal $(\mathrm{r}=-0.59)$. Otro estudio mostró la relación entre factores psicosociales asociados al agotamiento laboral (burnout) y al entusiasmo en enfermeras de un hospital público en México, en este estudio se identificó una correlación positiva entre el control laboral y el apoyo social $(\mathrm{r}=0.292)^{25}$.

Cabe mencionar, que el presente proyecto de investigación corresponde a un segundo documento que da seguimiento a una línea de trabajo, en la jurisdicción sanitaria número uno de los Servicios de Salud de Morelos ${ }^{1}$, en la cual se evaluó con antelación el apoyo social, el estrés percibido y el burnout.

Sin embargo, en México, no existe evidencia de la inclusión del personal de enfermería en estudios relacionados con un modelo de trayectorias, que considere la correlación entre factores psicosociales (demanda psicológica, control laboral y apoyo social), estrés psicológico y las dimensiones del burnout (ilusión por el trabajo, desgaste psíquico, indolencia y culpa).

El objetivo del trabajo fue evaluar la correlación entre factores psicosociales, el estrés psicológico, y las dimensiones del Síndrome de Burnout en un modelo de trayectorias en el personal de enfermería mexicano.

Hipótesis formulada: el modelo de trayectorias expresa las relaciones hipotéticas entre los factores psicosociales, el estrés psicológico y las dimensiones del Síndrome de Quemarse por el Trabajo.

\section{Material y Método}

Estudio transversal, observacional, analítico, llevado a cabo durante el mes de junio de 2012; población 397 profesionales de enfermería que laboraban en las jurisdicciones sanitarias número dos y tres de los Servicios de Salud del Estado de Morelos, la muestra fue seleccionada por conveniencia, conformada por 357 profesionales de enfermería, estuvo constituida por el $90 \%$ de la población en estudio. La participación del personal fue voluntaria, individual y anónima. Los criterios de inclusión fueron los siguientes: personal que laboraba en los Servicios de Salud de Morelos; adscrito a las jurisdicciones sanitarias número dos y tres; que asistió a la reunión en dónde se aplicaron las cédulas; que aceptó participar voluntariamente en el proyecto de investigación. Los criterios de exclusión fueron: personal adscrito a la jurisdicción sanitaria número uno; personal que no asistió a la reunión en dónde se aplicaron las cédulas; personal que no aceptó participar voluntariamente en el presente proyecto. 
Se aplicaron tres instrumentos de evaluación: Escala de Estrés Percibido (PSS por sus siglas en inglés) de Cohen ${ }^{26}$; el Cuestionario del Contenido del Trabajo (JCQ) ${ }^{11}$; y el Cuestionario para la Evaluación del Síndrome de Quemarse por el Trabajo (CESQT-PS) ${ }^{27}$.

La versión en español de la Escala de Estrés Percibido de Cohen, Kamarak, y Mermelstein ${ }^{26}$, traducida por Eduardo Remor y José Antonio Carrobles, está formada por 14 ítems, a favor del control (7 ítems; p. ej. Con qué frecuencia has sentido que tienes el control de todo) y pérdida de control (7 ítems; p. ej. Con qué frecuencia has estado enfadado / a porque las cosas que te han ocurrido estaban fuera de tu control). Los ítems se evalúan mediante una escala de frecuencia de 5 grados que va de 1 (Nunca) a 5 (Muy frecuentemente: todos los días), que corresponde a un caso igual a 3 (De vez en cuando) en la puntuación total. En el factor a favor del control las puntuaciones de los ítems 4-7, 9, 10, y 13, se invierten para valorar el estrés.

Se utilizó el Cuestionario del Contenido del Trabajo (JCQ por sus siglas en inglés) de Karasek ${ }^{11}$. Incluye las variables de demandas psicológicas ( 5 ítems; p. ej. Se me pide que realice una cantidad excesiva de trabajo), utilización de habilidades (6 ítems; p. ej. Mi trabajo requiere de un alto nivel de habilidad), autoridad de decisión ( 3 ítems; p. ej. En mi trabajo puedo tomar muchas decisiones por mí mismo), apoyo social de compañeros (4 ítems; p. ej. Mis compañeros de trabajo ayudan a que el trabajo se realice) y apoyo social del jefe (4 ítems; p. ej. Mi jefe se preocupa del bienestar del personal a su cargo). Los ítems se evalúan mediante una escala de frecuencia de 4 grados, que va de 1 (No, Totalmente en desacuerdo) a 4 ( $\mathrm{Si}$, Completamente de acuerdo), que concierne a un caso de valores iguales a 2 (En desacuerdo) y 3 (Estoy de acuerdo), en la puntuación total.

El Cuestionario para la Evaluación del Síndrome de Quemarse por el Trabajo (CESQT-PS), consta de 20 ítems que se distribuyen en cuatro subescalas denominadas: 1. Ilusión por el trabajo ( 5 ítems; p. ej. Veo mi trabajo como una fuente de realización personal); 2. Desgaste psíquico (4 ítems; p. ej. Me siento desgastada/o emocionalmente); 3. Indolencia (6 ítems; p. ej. No me apetece atender a algunos pacientes) y; 4 . Culpa (5 ítems; p. ej. Tengo remordimientos por algunos de mis comportamientos en el trabajo). Los ítems se evalúan mediante una escala de frecuencia de 5 grados, que va de 0 (Nunca) a 4 (Muy frecuentemente: todos los días).

En cuanto a la escala de medición, las variables en estudio tienen el siguiente rango: demanda psicológica 12-48; control laboral 24-96; apoyo social 8-32; ilusión en el trabajo 0-4; desgaste psíquico 0-4; indolencia 0-4; culpa 0-4, y estrés psicológico 1-5.

Cabe mencionar, que la Escala de Estrés Percibido, el Cuestionario del Contenido del Trabajo y el Cuestionario para la Evaluación del Síndrome de Quemarse por el Trabajo, son instrumentos válidos y confiables.

Para valorar el modelo estructural propuesto se consideraron diversos índices. El índice de bondad de ajuste (GFI), mide la cantidad relativa de varianza explicada por el modelo. El índice de ajuste no normado (NNFI), es un indicador del ajuste relativo del modelo. El índice de ajuste comparativo (CFI), indica el grado de ajuste del modelo cuando se compara con un modelo nulo. Para estos índices valores cercanos a 0.90 se consideran indicadores de un ajuste aceptable del modelo. La raíz cuadrada media del error de aproximación (RMSEA), estima la cantidad global de error existente en el modelo, valores entre 0.03 y 0.09 indican un ajuste adecuado del modelo. El índice de ajuste de parsimonia normada (PNFI), evalúa el grado de complejidad del modelo. Valores superiores a 0.50 se consideran adecuados cuando el índice de ajuste (NNFI) presenta valores cercanos a 0.90. Con relación a la consistencia interna de los instrumentos, valores superiores a 0.60 indican una confiabilidad adecuada.

En cuanto a la aplicación de la encuesta, se capacitó a personal externo para aplicar los tres instrumentos, no se les mencionó la hipótesis del estudio, el tiempo estimado para completar la encuesta fue de 30 minutos, su función fue explicar con detalle el propósito del estudio al personal de enfermería participante.

Para el análisis de resultados se utilizaron estadísticas descriptivas mediante la utilización del programa SPSS versión 19. El modelo de ecuaciones estructurales (MSE), se estimó por Máxima Verosimilitud (ML) e independencia de los residuos, se empleó el programa LISREL versión 8.30. Para el cálculo de 


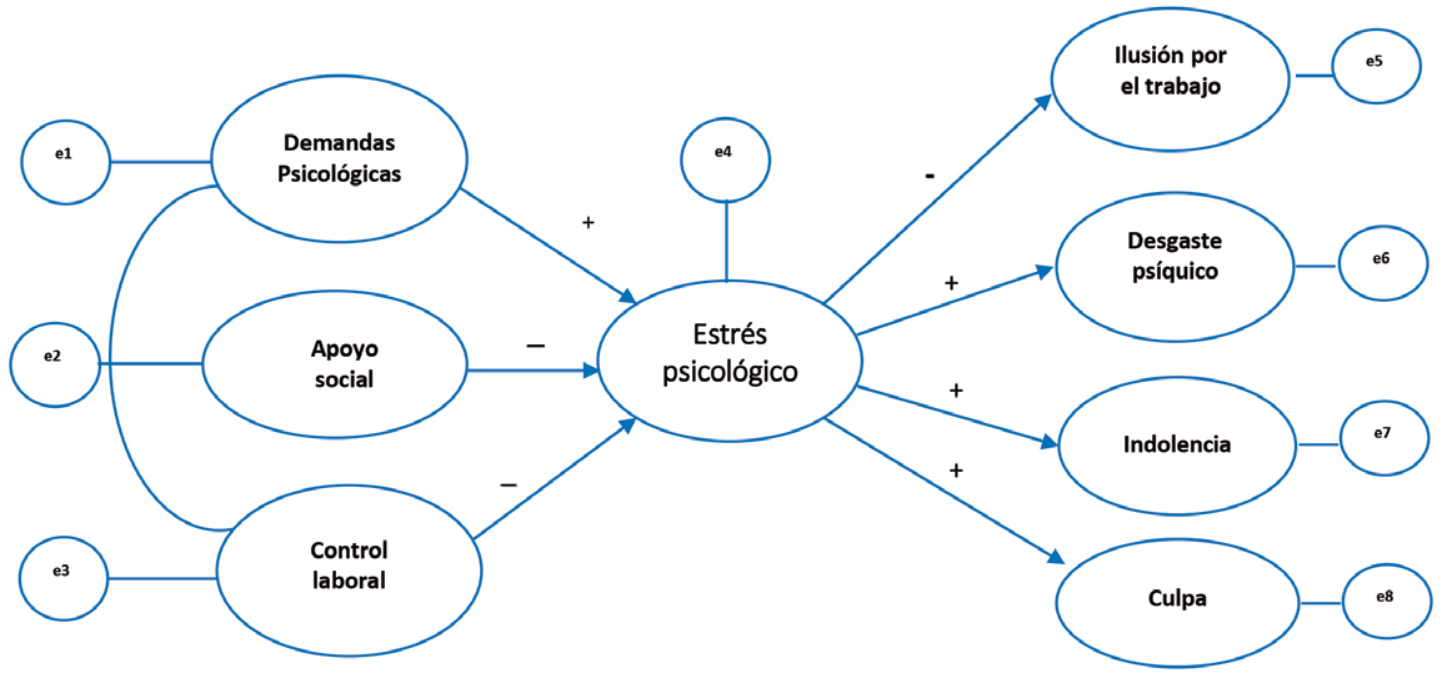

Figura 1. Diseño teórico de las direcciones y cargas esperadas del modelo. Fuente: Brito et al'.

la consistencia interna se usó el coeficiente alfa de Cronbach; y la validez concurrente por medio de la correlación lineal de Pearson (r). El modelo teórico se aprecia en la figura 1.

Aspectos éticos, el presente proyecto de investigación se desarrolló con el consentimiento previo de los participantes. Se solicitó la autorización del Comité de Ética de la Subdirección de Enseñanza, Capacitación e Investigación de los Servicios de Salud de Morelos (SSM), para efectuar este estudio.

\section{Resultados}

La muestra consistió en 357 profesionales de enfermería, el 98\% correspondió al sexo femenino y el 2\% al masculino; la edad promedio encontrada fue de 39 años con una desviación estándar de 10.2, con un rango de 23 a 72 años. En cuanto a la formación académica en enfermería, se tiene un predominio de personal técnico $(77 \%)$, seguido de licenciados $(15 \%)$ y por último auxiliares $(8 \%)$.

En el cuadro 1 se presentan los estadísticos descriptivos de las variables. En cuanto a las medias, se registran niveles bajos de demandas psicológicas, niveles altos de control laboral, de apoyo social y de ilusión por el trabajo, niveles bajos de indolencia y culpa, niveles moderados de desgaste psíquico y estrés psicológico. Las variables mostraron niveles adecuados de confiabilidad, con excepción de la demanda psicológica.

Cuadro 1. Estadísticos descriptivos de las variables psicométricas de estudio $(n=357)$

\begin{tabular}{lccrl}
\hline Variables & Rango & Media & \multicolumn{1}{c}{ DE } & Alfa de Cronbach \\
\hline Factores psicosociales & & & & 0.73 \\
\hline Demanda psicológica & $12-48$ & 29.86 & 6.09 & 0.53 \\
\hline Control laboral & $24-96$ & 77.45 & 11.33 & 0.73 \\
\hline Apoyo social & $8-32$ & 23.86 & 4.46 & 0.79 \\
\hline Burnout (SQT) & & & & 0.67 \\
\hline Ilusión en el Trabajo & $0-4$ & 3.54 & 0.62 & 0.62 \\
\hline Desgaste Psíquico & $0-4$ & 1.37 & 0.83 & 0.75 \\
\hline Indolencia & $0-4$ & 0.56 & 0.49 & 0.66 \\
\hline Culpa & $0-4$ & 1.01 & 0.72 & 0.74 \\
Estrés psicológico & $1-5$ & 2.80 & 0.59 & 0.82 \\
\hline
\end{tabular}


Correlación bivariada entre apoyo social, estrés percibido y burnout

De acuerdo con las correlaciones en el cuadro 2, el apoyo social se asoció de manera positiva con el control laboral ( $\mathrm{r}=0.395, \mathrm{p} \leq .01)$, y negativa con las demandas psicológicas $(\mathrm{r}=-0.129, \mathrm{p} \leq 0.05)$; el estrés se relacionó positivamente con las demandas psicológicas $(\mathrm{r}=0.107, \mathrm{p} \leq 0.05)$. Al analizar las relaciones del estrés con las dimensiones del burnout se observó una correlación positiva entre el estrés con el desgaste psíquico $(\mathrm{r}=0.236, \mathrm{p} \leq 0.01)$, la indolencia $(\mathrm{r}=0.156, \mathrm{p} \leq 0.01)$ y la culpa $(\mathrm{r}=0.145, \mathrm{p} \leq 0.01)$; es relevante mencionar, la relación positiva entre desgaste psíquico con la indolencia $(\mathrm{r}=0.411, \mathrm{p} \leq 0.01)$ y la culpa $(\mathrm{r}=0.297, \mathrm{p} \leq 0.01)$; así también, la relación positiva entre la indolencia con el sentimiento de culpa $(\mathrm{r}=0.451, \mathrm{p} \leq 0.01)$.

Cuadro 2. Matriz de correlaciones entre las variables psicométricas de estudio $(n=357)$

\begin{tabular}{|c|c|c|c|c|c|c|c|c|}
\hline Variables & 1 & 2 & 3 & 4 & 5 & 6 & 7 & 8 \\
\hline 1. Demandas psicológicas & 1 & & & & & & & \\
\hline 2. Control laboral & -0.027 & 1 & & & & & & \\
\hline 3. Apoyo social & $-0.129 *$ & $0.395 * *$ & 1 & & & & & \\
\hline 4. Estrés psicológico & $0.107 *$ & -0.057 & -0.052 & 1 & & & & \\
\hline 5. Ilusión por el Trabajo & -0.037 & $0.188 * *$ & 0.091 & -0.082 & 1 & & & \\
\hline 6. Desgaste Psíquico & $0.335 * *$ & $-0.044 * *$ & $-0.230 * *$ & $0.236 * *$ & $-0.217 * *$ & 1 & & \\
\hline 7. Indolencia & $0.127 *$ & $-0.137 * *$ & $-0.133 *$ & $0.156 * *$ & $-0.330 * *$ & $0.411 * *$ & 1 & \\
\hline 8. Culpa & 0.013 & -0.097 & -0.093 & $0.145 * *$ & $-0.174 * *$ & $0.297 * *$ & $0.451 * *$ & 1 \\
\hline
\end{tabular}

Modelo de trayectorias: factores psicosociales, estrés percibido y burnout

El modelo de trayectorias representado en el cuadro 3, obtuvo un ajuste adecuado, excepto con el GFI que presentó un valor inferior a 0.90 , lo que significa que 0.54 es la cantidad relativa de varianza explicada por el modelo.

Cuadro 3. Índices de ajuste global para el modelo de trayectorias

\begin{tabular}{llllllll}
\hline $\mathrm{Chi}^{2}$ & $\mathrm{Gl}$ & $\mathrm{P}$ & $\mathrm{GFI}^{\mathrm{a}}$ & $\mathrm{RMSEA}^{\mathrm{b}}$ & $\mathrm{NNFI}^{\mathrm{c}}$ & $\mathrm{CFI}^{\mathrm{d}}$ & $\mathrm{PNFI}^{\mathrm{e}}$ \\
8414 & 1474 & 0.000 & 0.54 & 0.07 & 0.90 & 0.90 & 0.76 \\
\hline
\end{tabular}

${ }^{\mathrm{a}} \mathrm{GFI}=$ Goodness Fit Index, ${ }^{\mathrm{b}}$ RMSE $A=$ Root Mean Square Error of Approximation, ${ }^{\mathrm{c}}$ NNFI $=$ Non-Normed Fit Index,

${ }^{\mathrm{d}} \mathrm{CFI}=$ Comparative Fit Index, ${ }^{\text {ePNFI }}=$ Parsimony Normed Fit Index.

Efectos directos los factores psicosociales, el estrés percibido y las dimensiones del burnout.

En el cuadro 4 se incluyen los efectos directos de los factores psicosociales en el trabajo con el estrés percibido, asimismo, los efectos directos del estrés percibido con las dimensiones del burnout. En este análisis es importante resaltar que la demanda psicológica, el control laboral y el apoyo social manifestaron tener un peso directo moderado sobre el estrés psicológico $(0.19,-0.21$ y -0.25 , respectivamente). Por otra parte, el estrés psicológico manifestó tener una influencia directa elevada sobre la ilusión en el trabajo (-0.40), el desgaste psíquico (0.68), la indolencia (0.64) y la culpa (0.53).

En términos específicos puede observarse que el estrés psicológico, denota un peso importante como variable mediadora entre los factores psicosociales y las dimensiones del SQT.

En la figura 2 se puede observar la varianza explicada entre las variables del modelo estructural. La varianza explicada del estrés por el moderado nivel de demanda psicológica, el elevado nivel de control laboral y el elevado apoyo social fue de 0.11 . La varianza explicada por la ilusión fue de 0.16 , la culpa correspondió a 0.28 , la indolencia a 0.41 y para el desgaste psíquico fue de 0.47 . 
Cuadro 4. Efectos directos de las variables demanda psicológica, estrés y burnout ( $n=357)$

\begin{tabular}{llc}
\hline Variables & Efecto directo & Puntaje \\
\hline Demanda psicológica & Estrés psicológico & 0.19 \\
\hline Control laboral & Estrés psicológico & -0.21 \\
\hline Apoyo social & Estrés psicológico & -0.25 \\
\cline { 2 - 3 } Estrés psicológico & Ilusión en el trabajo & -0.40 \\
\cline { 2 - 3 } & Desgaste psíquico & 0.68 \\
\cline { 2 - 3 } & Indolencia & 0.64 \\
\cline { 2 - 3 } & Culpa & 0.53 \\
\hline
\end{tabular}

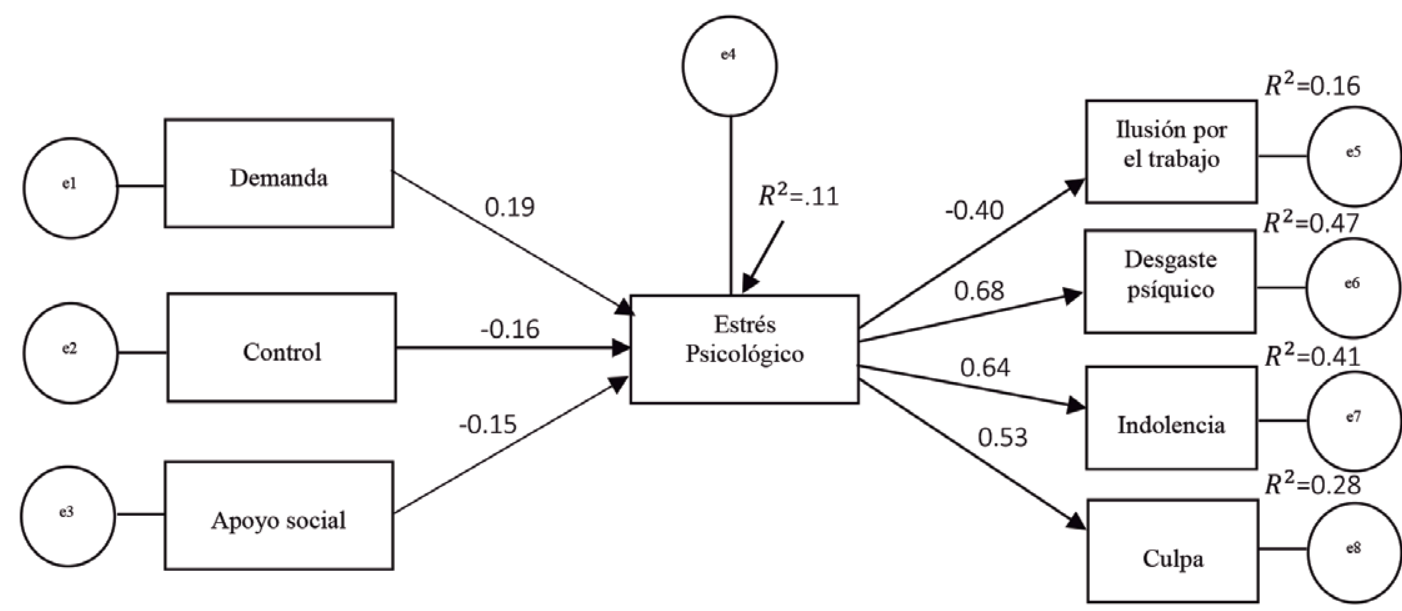

Figura 2. Representación gráfica del modelo de trayectorias sobre factores psicosociales, estrés psicológico y burnout

Al comparar las relaciones del modelo de trayectorias con los resultados obtenidos se analiza que la demanda psicológica incrementa el estrés psicológico; además, los altos niveles de control laboral disminuyen la percepción de estrés; por otra parte, el elevado nivel de apoyo social disminuye el estrés psicológico. Se observa que el estrés percibido disminuye la ilusión por el trabajo e incrementa el desgaste psíquico, la indolencia y la culpa, resultados esperados teóricamente.

\section{Discusión y conclusiones}

En los resultados se presentan las evidencias del modelo de trayectorias conformado por las variables latentes: demanda psicológica, control laboral, apoyo social, estrés psicológico; ilusión por el trabajo, desgaste psíquico, indolencia y culpa; dichas evidencias aún son escasas en nuestro país y en Latinoamérica, el presente estudio tuvo por objeto abonar a dichas evidencias empíricas.

En nuestro estudio se encontraron niveles moderados de demandas psicológicas (29.86), resultado inferior al reportado en el estudio de Juárez ${ }^{14}$ que correspondió a 30.04 y en un estudio realizado en algunos países latinoamericanos como Chile (30.38), México (30.34) y Colombia (31.98) ${ }^{28}$. En relación con el apoyo social, los niveles fueron altos (23.86), resultado superior al reportado en el estudio latinoamericano, [Chile (23.72), México (21.99) y Colombia (20.91)].

El nivel de la dimensión control presentó un alto nivel (77.45) resultado mayor que el identificado en países latinoamericanos, en Chile (63.74), México (72.85) y Colombia (61.88).

Es importante señalar, que el personal de enfermería en estudio, percibió niveles moderados de estrés psicológico (2.80), resultado similar al identificado en Morelos, México (2.40).

Sobre las dimensiones del burnout, se identificó una relación negativa entre la ilusión por el trabajo con el desgaste psíquico, con la indolencia y con la culpa; se identificó una relación positiva entre el desgaste psíquico, con la indolencia y con la culpa, lo que es consistente con la literatura empleada. 
En el presente estudio, los niveles de ilusión por el trabajo fueron altos (3.54), caso contrario, se presentaron bajos niveles de desgaste psíquico (1.37), de indolencia (.56) y de culpa (1.01), lo que significa que el personal de enfermería no se encuentra sometido a factores demandantes.

Por otra parte, si se tiene en cuenta la relación que guarda el estrés psicológico con las cuatro dimensiones de CESQT-PS, al aumentar el nivel del estrés psicológico el valor de la ilusión por el trabajo disminuyó. Por el contrario, al aumentar el estrés psicológico percibido por el personal de enfermería aumentó el nivel del desgaste psíquico, la indolencia y la culpa, estos resultados son similares a los encontrados por Mercado-Salgado ${ }^{24}$ quien desarrolló un estudio relacionado con el estrés y el desgaste profesional (burnout), en personal de enfermería de un hospital materno infantil de atención a la población abierta, si bien en este estudio se utilizaron instrumentos diferentes para evaluar el burnout y el estrés, los resultados son similares al presente documento, el nivel de estrés identificado fue moderado; el personal de enfermería no manifestó síntomas de desgaste profesional. Al aumentar el nivel del estrés la realización personal disminuyó. Por el contrario, al incrementar el estrés aumentó el nivel de las dimensiones del burnout: el cansancio emocional y la despersonalización.

El apoyo social se manifestó como un factor asociado con el control laboral, este resultado fue similar al encontrado por Juárez ${ }^{28}$, quien identificó una correlación positiva entre el apoyo social y el control laboral.

Sobre la hipótesis formulada el modelo de trayectorias expresa las relaciones hipotéticas entre los factores psicosociales, el estrés psicológico y las dimensiones del síndrome de quemarse por el trabajo, resultó aceptada.

De acuerdo a todos los datos que hemos venido aportando en el presente proyecto de investigación, además de tener en cuenta los alcances obtenidos, concluimos al retomar el modelo de trayectorias, que el estrés es un fenómeno psíquico influido por una serie de factores del ambiente que son el input del proceso, para que el estrés se produzca y el individuo evalúe su capacidad y recursos para afrontarlo. El moderado grado de estrés percibido por el personal de enfermería en la muestra de estudio, estuvo antecedido por la presencia de un nivel moderado de demandas psicológicas, altos niveles de control, altos niveles de apoyo social; esto generó altos niveles de ilusión por el trabajo, y bajos niveles de desgaste psíquico, indolencia y sentimientos de culpa.

\section{Limitaciones del Estudio}

Nuestro estudio tiene limitaciones en el diseño, es importante puntualizar, que no se tuvieron datos suficientes para determinar estadísticamente la dirección causal de las relaciones estudiadas entre la demanda psicológica, el control en el trabajo, el apoyo social, el estrés psicológico, y el burnout. La muestra no fue aleatoria respecto al personal de enfermería del Estado de Morelos, sino de conveniencia en dos de tres jurisdicciones sanitarias de los Servicios de Salud de Morelos; una vez que la confiabilidad de los instrumentos, el nivel de la variable demanda psicológica fue bajo (0.53). Con respecto al modelo factorial confirmatorio, la cantidad relativa de varianza explicada por el modelo alcanzó un valor de 54\%.

\section{Responsabilidades éticas}

Protección de personas y animales: Los autores declaran que para esta investigación no se realizaron experimentos en seres humanos ni en animales.

Confidencialidad de los datos: Se resguardo de forma cuidadosa la identidad, confidencialidad y privacidad de cada uno de los participantes.

Derecho a la privacidad y consentimiento informado: Los autores han obtenido el consentimiento informado de los participantes. Este documento obra en poder del autor de correspondencia.

Financiamiento: Ninguno.

Conflicto de intereses: Los autores declaran no tener ningún conflicto de intereses. 


\section{Referencias}

1. Brito-Ortíz JF, Nava-Gomez ME, Juárez-García A. Un modelo estructural de las relaciones entre apoyo social, estrés percibido y burnout en enfermeras mexicanas Psicología y Salud. 2015; 25(2):157-67.

2. Gil-Monte PR, García-Juesas JA, Caro-Hernández M. Influencia de la sobrecarga laboral y la autoeficacia sobre el Síndrome de Quemarse por el Trabajo (burnout) en profesionales de enfermería. R. interim. Psico 2008; 42(1):113-8.

3. Brito-Ortíz JF, Nava-Gómez ME, Brito-Nava E, Juárez-García A, López M. Estrés percibido y desempeño laboral en enfermeras: un análisis estructural. Rev Mex Sal Trab 2016; 7-8 (17-18): 10-7.

4. Nabirye RC, Brown KC, Pryor ER, Maples EH. Occupational stress, job satisfaction and job performance among hospital nurses in Kampala, Uganda. J Nurs Manag. 2011; 19(6):760-8. https://doi.org/10.1111/j.1365-2834.2011.01240.x

5. Cooper CL. Capítulo 9 Reacciones al estrés en los trabajadores manuales y no manuales. En: Kalimo R, El-Batawi MA, Cooper CL. Los factores psicosociales en el trabajo y su relación con la salud. Ginebra: OMS 1988. p. 94

6. Moreno-Jiménez B, Báez-León C. Factores y riesgos psicosociales, formas, consecuencias, medidas y buenas prácticas. NIPO 792-11-088-1. Madrid: MITRAMISS/INSHT/Universidad Autónoma de Madrid2010.

7. Freimann T, Merisalu E. Work-related psychosocial risk factors and mental health problems amongst nurses at a university hospital in Estonia: a cross-sectional study. Scand J Public Health 2015; 43(5): 447-52. https://doi.org/10.1177/1403494815579477

8. Toh SG, Ang E, Devi MK. Systematic review on the relationship between the nursing shortage and job satisfaction, stress and burnout levels among nurses in oncology/haematology settings. Int J Evid Based Healthc. 2012; 10(2): 126-41. https://doi.org/10.1111/j.1744-1609.2012.00271.x

9. Leka S, Jain A. Health impact of psychosocial hazards at work: an overview. Geneva: World Health Organization. 2010. p. 126

10. Gil-Monte PR. Algunas razones para considerar los riesgos psicosociales en el trabajo y sus consecuencias en la salud pública. Rev Esp Salud Pública. 2009; 83(2): 169-73.

11. Karasek RA. Job demands, job decision latitude, and mental strain: Implications for job redesign. Adm Sci Q 1979; 24(2): 285-308. https://doi.org/10.2307/2392498

12. Shen H-C, Cheng Y, Tsai P-J, Lee SS, Guo YL. Occupational stress in nurses in psychiatric institutions in Taiwan. J Occup Health. 2005; 47 (3): 218-25. https://doi.org/10.1539/joh.47.218

13. Brown DE, James GD, Mills PS. Occupational differences in job strain and physiological stress: female nurses and school teachers in Hawaii. Psychosom Med 2006; 68(4): 524-30. https://doi.org/10.1097/01.psy.0000222356.71315.8e

14. Juárez-García A. Factores psicosociales relacionados con la salud mental en profesionales de servicios humanos en México. Cienc. trab. 2004; 6(14): 189-96.

15. Juárez-García A. Factores psicosociales laborales relacionados con la tensión arterial y síntomas cardiovasculares en personal de enfermería en México. Salud pública Mex. 2007; 49(2): 109-17.

16. Gil-Monte PR. El síndrome de quemarse por el trabajo (burnout). Una enfermedad laboral en la sociedad del bienestar. Madrid: Pirámide; 2005.

17. Ratto-Dattoli A, García-Pérez RC, Silva MI, González MC. El Síndrome de Quemarse por el Trabajo y factores psicosociales en docentes de primaria de la ciudad de Montevideo. Cienc. psicol.. 2015; 9(2): 273-81.

18. Gil-Monte PR, Unda-Rojas S, Sandoval-Ocaña JI. Validez factorial del $<<$ Cuestionario para la Evaluación del Síndrome de Quemarse por el Trabajo>> (CESQT) en una muestra de maestros mexicanos. Salud Ment. 2009; 32(3): 205-14.

19. Trifiletti E, Pedrazza M, Berlanda S, Pyszczynski T. Burnout disrupts anxiety buffer functioning among nurses: A three-way interaction model. Front. Psychol. 2017; 8:1362. 
https://doi.org/10.3389/fpsyg.2017.01362

20. Brady M. Death anxiety among emergency care workers. Emerg Nurse. 2015; 23(4): 32-7. https://doi.org/10.7748/en.23.4.32.e1448

21. Yang T, Shen Y-M, Zhu M, Liu Y, Deng J, Chen Q, et al. Effects of co-worker and supervisor support on job stress and presenteeism in an aging workforce: a structural equation modelling approach. Int J Environ Res Public Health. 2015; 13(1): e15. https://doi.org/10.3390/ijerph13010072

22. Amutio-Kareaga A. Afrontamiento del estrés en las organizaciones: un programa de manejo a nivel individual/grupal. Rev. Psicol. trab. organ. 2004; 20(1): 77-93.

23. Cañadas-De la Fuente GA, Vargas C, San Luis C, García I, Cañadas GR, De la Fuente EI. Risk factors and prevalence of burnout syndrome in the nursing profession. Int J Nurs Stud. 2015; 52(1): 240-9. https://doi.org/10.1016/j.ijnurstu.2014.07.001

24. Mercado-Salgado S. Estrés y desgaste profesional (burnout) en personal de enfermería de un hospital materno infantil de atención a la población abierta. Administración contemporánea, Revista de Investigación. 2007; 1 (7): e26.

25. Juárez-García A. Factores psicosociales asociados al agotamiento laboral (Burnout) y al entusiasmo (engagement) en enfermeras de un hospital público en México. En Arias-Galicia F, Juárez-García A. Agotamiento profesional y estrés, hallazgos desde México y otros países latinoamericanos. Ciudad de México: Porrúa; 2012. p. 125-53.

26. Cohen S, Kamarck T, Mermelstein R. A global measure of perceived stress. J Health Soc Behav. 1983; 24(4): 385-96. https://doi.org/10.2307/2136404

27. Gil-Monte PR. Manual CESQT, Cuestionario para la Evaluación del Síndrome de Quemarse por el Trabajo. Madrid: TEA Ediciones; 2011.

28. Juárez-García A, Vera-Calzaretta A, Merino-Soto C, Gómez-Ortíz V, Feldman L, HernándezMendoza E. Demanda/control y la salud mental en profesionales de la salud: un estudio en seis países latinoamericanos. Inform. psicol. 2014; (108): 2-18. http://dx.medra.org/10.14635/ IPSIC.2014.108.1 